\title{
A simple process for the fabrication of large-area CVD graphene based devices via selective in-situ functionalization and patterning
}

\author{
Arseny M Alexeev, Matthew D Barnes, V Karthik Nagareddy, \\ Monica F Craciun, and C David Wright \\ Centre for Graphene Science, CEMPS, University of Exeter, Exeter EX4 4QL, UK \\ E-mail: david.wright@exeter.ac.uk
}

\begin{abstract}
We report a novel approach for the fabrication of micro- and nano-scale graphene devices via the in-situ plasma functionalization and in-situ lithographic patterning of large-area graphene directly on CVD catalytic metal $(\mathrm{Cu})$ substrates. This enables us to create graphene-based devices in their entirety prior to any transfer processes, simplifying very significantly the device fabrication process and potentially opening up the route to the use of a wider range of target substrates. We demonstrate the capabilities of our technique via the fabrication of a flexible, transparent, graphene/graphene oxide humidity sensor that outperforms a conventional commercial sensor.
\end{abstract}

PACS numbers: 68.65.Pq, 81.05.ue

Keywords: Graphene, Oxygen Plasma, Functionalization, Nanopattering, Humidity Sensing

\section{Introduction}

Graphene has attracted huge interest in recent years due to its potential for delivering novel and high-performance electronic and optoelectronic devices. Progress in the production of large-area mono- and few-layer graphene by chemical vapor deposition (CVD) techniques has brought the practical realization of graphene devices closer to reality. However, the efficient and cost-effective production of graphene devices is still held back by conventional approaches to device fabrication. These involve several process steps including graphene film transfer, ex-situ lithographic patterning and metal contact deposition; processes that are time consuming, not always reproducible and potentially deleterious to the properties of the CVD-graphene layer $[1,2,3,4,5,6,7]$. In this paper we demonstrate a new and attractive approach to the fabrication of microand nano-scale graphene devices that combines the in-situ plasma functionalization 
and in-situ lithographic patterning of large-area CVD graphene directly on copper substrates, allowing for the fabrication of devices in their entirety prior to any transfer steps. We demonstrate our technique by producing, entirely in-situ on $\mathrm{Cu}$ foils, a graphene/graphene-oxide based humidity sensor that outperforms a conventional commercial sensor.

Significant progress has been made in recent times towards controlling the electronic, optical and mechanical properties of graphene via functionalization with a number of chemicals including fluorine $[8,9,10,11,12]$, chlorine $[13,14]$, ferric chloride $[15,16,17]$, and, as used in this paper, oxygen [18, 19] having all successfully been demonstrated. The attachment of oxygen radicals during plasma exposure progressively transforms graphene's band structure, creating a bandgap [18, 19, 20] and producing insulating graphene oxide (GO) [21, 22, 23, 24]. GO-based devices have widespread and important applications, including, for example, non-volatile memories [25, 26], memristors [27], supercapacitors [28], gas sensors [29], and humidity sensors [30, 31].

The plasma oxidation method for the production of graphene oxide is advantageous over liquid-phase fabrication, since it does not contaminate samples with by-products of (wet) chemical reactions [19, 20, 23]. It is also scalable, environment-friendly and involves fewer stages as compared to conventional wet-chemical methods [32, 33, 34]. Patterned graphene/graphene oxide structures and devices can also be created using plasma modification of graphene combined with lithographic processing. Here we report on a novel, large-area-compatible method for the in-situ, pre-transfer, fabrication of such graphene/graphene-oxide devices on CVD (catalytic) $\mathrm{Cu}$ foils. In this way complex micro- and nano-scale GO device structures can be reliably fabricated in fewer steps than via conventional approaches and, importantly, be readily transferred onto a range of target surfaces including rigid and flexible substrates and possibly even textiles. Device fabrication via plasma oxidation post-transfer (the conventional approach) would be more limiting in the choice of target substrates, since many substrates would be damaged/degraded by the plasma bombardment process.

\section{Results and discussion}

Graphene films used in this study were grown using a commercial cold-wall nanoCVD system in which catalytic metal foils are resistively heated inside a CVD chamber [35]. Selective heating of only a small area of the CVD chamber and keeping the wall temperature below $100^{\circ} \mathrm{C}$ reduces both the chamber contamination by growth products and the energy consumption (cf. conventional CVD systems). The cold-wall CVD growth also benefits from faster heating and cooling rates, shorter growth times, and reduced gas usage $[36,37]$. (A comprehensive description of the CVD growth of our samples, together with the post-growth characterization data, is included in the Methods Section and Supplementary Material).

Our as-grown CVD films consist of monolayer graphene crystals which are less than $1 \mu \mathrm{m}$ in size, with grain boundaries formed between individual domains. Some isolated 
(a)



(b)

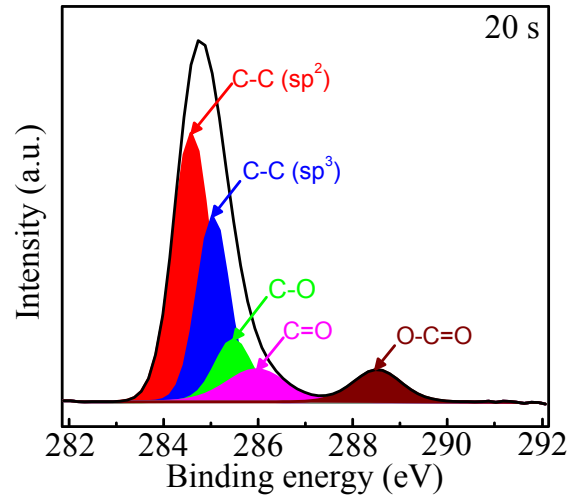

(c)

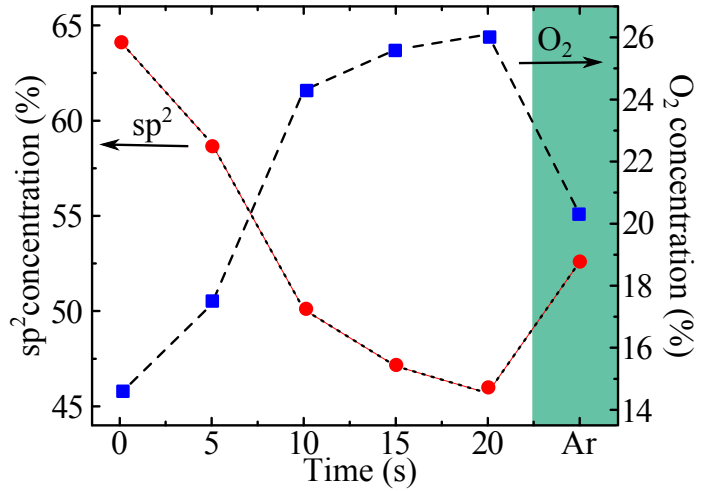

(d)

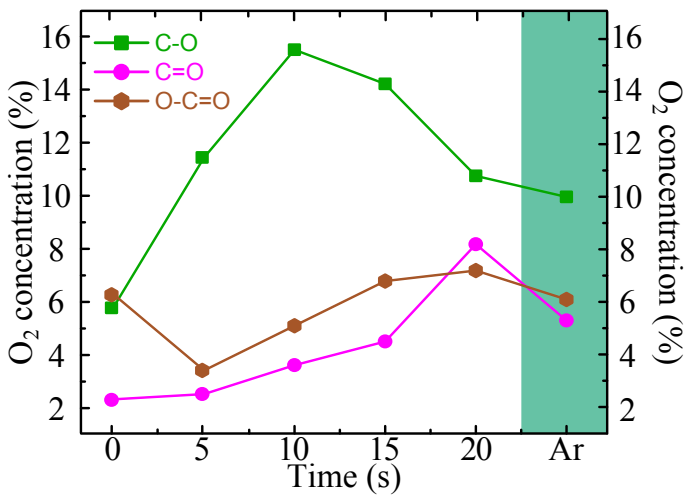

Figure 1. (Colour online) High-resolution XPS C1s spectra of (a) unoxidized CVD graphene film and (b) after 20s of plasma modification. Effect of plasma treatment time on (c) total concentration of $\mathrm{sp}^{2}$ and oxygen and (d) individual chemical composition of oxygen functional groups. (Green background indicates results after thermal annealing in Ar atmosphere).

multilayer patches are also present in our samples, but cover an insignificant part (less than $1 \%$ ) of the whole film. Continuity of the films was confirmed by SEM imaging (see Supplementary Material (Figure S1)).

Graphene films on $\mathrm{Cu}$ foils were treated with oxygen plasma using a commercial reactive ion etching system. Samples were placed approximately $15 \mathrm{~cm}$ away from the plasma stream to avoid the graphene layer being damaged by UV radiation and high-energy ions [24]; oxygen radicals arrive at the graphene site energetically relaxed as confirmed by the (plasma) power-dependent Raman measurements provided in the Supplementary Material (Figure S4).

Results of the XPS measurements performed on the functionalized samples (while still on $\mathrm{Cu}$ foils) are presented in Figure 1. Treatment times in the range of $0-20 \mathrm{~s}$ were used to achieve differing levels of oxygen modification. Figure 1(a) shows the high-resolution XPS C1s spectra of un-oxidized CVD graphene film on a $\mathrm{Cu}$ foil. Deconvolution of the spectra showed two major peaks at $284.4 \mathrm{eV}$ and $284.8 \mathrm{eV}$, corresponding to $\mathrm{C}-\mathrm{C}\left(\mathrm{sp}^{2}\right)$ and $\mathrm{C}-\mathrm{C}\left(\mathrm{sp}^{3}\right)$ bonding and three shoulder peaks at 285.4 $\mathrm{eV}, 286.5 \mathrm{eV}$ and $288.3 \mathrm{eV}$ related to $\mathrm{C}-\mathrm{O}$ (epoxide), $\mathrm{C}=\mathrm{O}$ (carbonyl) and $\mathrm{O}-\mathrm{C}=\mathrm{O}$ (carboxyl) bonds, respectively $[24,38,39]$. The $\mathrm{sp}^{3}$ and oxygen contributions in the as- 
grown sample can be attributed to the presence of grain boundaries in the graphene film with reactive edge sites which attracts residual oxygen contaminants from the ambient air exposure, as reported previously [40,41]. After oxygen plasma treatment, the relative peak intensity of oxygen functional groups increased with time (Figure 1(b), also see Supplementary Material), indicating successful incorporation of oxygen species on the graphene surface. The increase in plasma treatment time also resulted in the increase in the $\mathrm{sp}^{3} /$ defect peak and an associated decrease in $\mathrm{sp}^{2}$ peak intensity. As oxygen radicals react with the graphene film, the $\mathrm{sp}^{2}$ symmetry is destroyed and more $\mathrm{sp}^{3}$ sites emerge; this corresponds to the increased level of oxygenated functional groups. The overall concentration of oxygen present on the graphene surface increased to approximately 26 at.\% after $20 \mathrm{~s}$ of oxygen plasma exposure (as shown in Figure 1(c), note saturation of oxygen uptake by the graphene film after around $10 \mathrm{~s}$ of oxidation). A closer inspection of individual functional species (summarized in Figure 1(d)) reveals a reduction of epoxide groups and a small increase in carbonyl and carboxyl functional groups between 10 and $20 \mathrm{~s}$ exposure. Here, the increase in $\mathrm{C}=\mathrm{O}$ and $\mathrm{O}-\mathrm{C}=\mathrm{O}$ groups indicates the rise in plasma-induced edge sites (damaging) in the graphene film as result of prolonged exposure to the oxygen plasma radicals.

Although, oxygen functional groups are relatively stable at room temperature, they can be in principle removed at elevated temperatures. Thermal reduction techniques, in either vacuum or Ar atmosphere, were employed previously to effectively remove oxygen groups from the graphene surface [20, 21, 42, 43]. In order to investigate the effect of thermal treatment on the chemical composition of our plasma-oxidized graphene films, we subjected them to temperature annealing at $220^{\circ} \mathrm{C}$ in $\mathrm{Ar}$ for 60 min. The deconvoluted XPS C1s spectra for a graphene film oxidized for 20s and then thermal annealed in this way is shown in Supplementary Material (Figure S2), with the results summarized in Figure 1(d). A significant decrease in the relative intensity of all oxygenated groups is seen. This reduction is accompanied by a partial recovery of $\mathrm{sp}^{2}$ cluster concentration (see Figure $1(\mathrm{c})$ ), demonstrating that our plasma processing approach is essentially reversible.

Structural changes in the graphene backbone upon plasma treatment were also investigated using Raman spectroscopy, with the results presented in Figure 2 (note that to exclude $\mathrm{Cu}$ photoluminescence background from the Raman signal, the graphene films were transferred onto $\mathrm{Si} / \mathrm{SiO}_{2}$ substrate for the Raman studies and that the Raman spectrum was normalised to the intensity of the Si peak). In Figure 2(a) we plot the Raman spectrum evolution against plasma exposure time. We see a continuous increase of the D peak intensity $\left(I_{\mathrm{D}}\right)$ with exposure time (since the XPS studies showed effective saturation of oxidation after $10 \mathrm{~s}$ of plasma exposure, we limited our Raman studies to this range), accompanied by a simultaneous decrease of the $\mathrm{G}\left(I_{\mathrm{G}}\right)$ and $2 \mathrm{D}\left(I_{2 \mathrm{D}}\right)$ peaks. The $I_{\mathrm{D}} / I_{\mathrm{G}}$ ratio is plotted in Figure $2(\mathrm{~b})$ and shows a monotonic increase with increasing plasma exposure time; this is an indication of the breaking of the symmetry of graphene $\mathrm{sp}^{2}$ bonds due to the attachment of oxygen species, as well as an indication of the formation of defects caused by the plasma [18, 19, 21, 22, 23, 24, 32, 33, 34, 38, 39]. The 
(a)

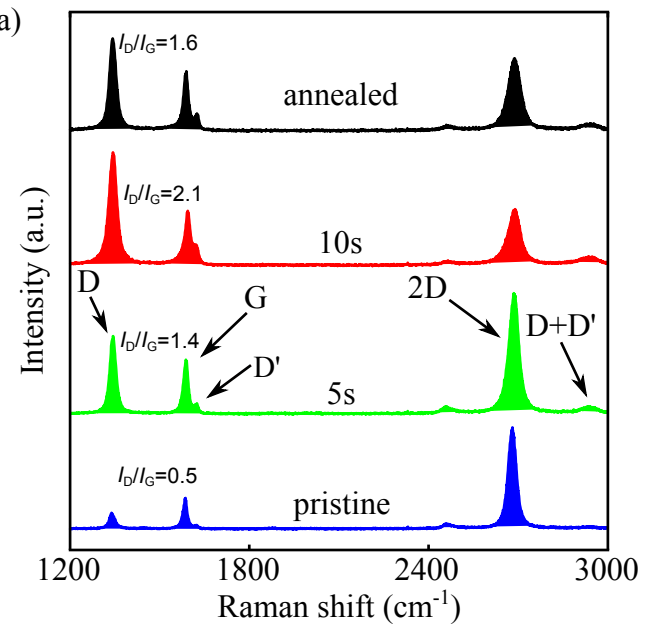

(b)

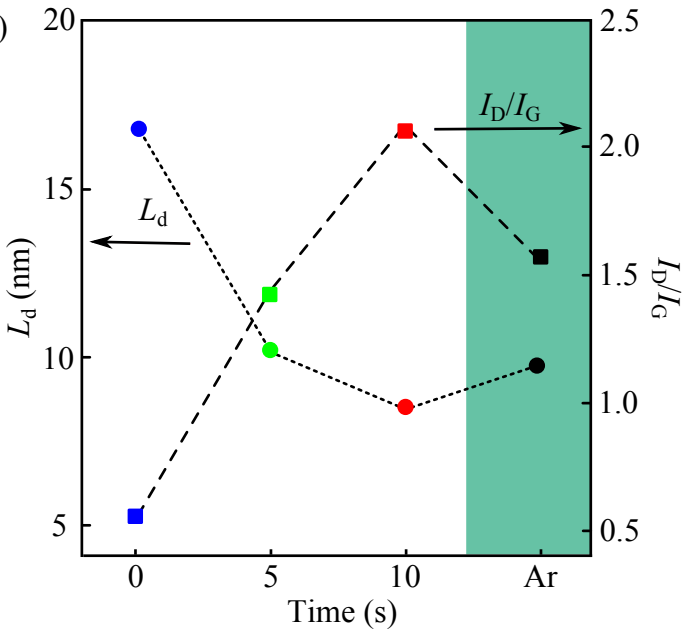

Figure 2. (Colour online) (a) Evolution of the CVD graphene Raman spectra upon functionalization by $\mathrm{O} 2$ plasma and after thermal annealing in Ar. (b) Raman D-to$\mathrm{G}$ peak intensity $\left(I_{\mathrm{D}} / I_{\mathrm{G}}\right)$ ratio and average distance between $\mathrm{sp}^{2}$ clusters $\left(L_{\mathrm{d}}\right)$ as a function of plasma exposure time. (Green background indicates results after thermal annealing in Ar atmosphere).

average inter-defect distance, $L_{\mathrm{d}}$, can be estimated using the Tuinstra-Koenig relation $[44,45], L_{\mathrm{d}}^{2}\left(\mathrm{~nm}^{2}\right)=\frac{4.3 \times 10^{3}}{E_{\mathrm{L}}^{4}\left(\mathrm{eV}^{4}\right)}\left[I_{\mathrm{G}} / I_{\mathrm{D}}\right]$, where $E_{\mathrm{L}}$ is the laser photon energy measured in $\mathrm{eV}$. The results of applying this equation to our Raman data are plotted in Figure 2(b); $L_{\mathrm{d}}$ decreases, from around $17 \mathrm{~nm}$ to around $8 \mathrm{~nm}$, with increasing treatment time, reflecting an increase in defect density during plasma functionalization.

Turning back to Figure 2(a), one can notice emergence of $\mathrm{D}^{\prime}$ and $\mathrm{D}+\mathrm{D}^{\prime}$ peaks (located at around $1600 \mathrm{~cm}^{-1}$ and $2940 \mathrm{~cm}^{-1}$ ) after $5 \mathrm{~s}$ of plasma exposure and the merging of $\mathrm{D}^{\prime}$ and $\mathrm{G}$ peaks after 10s. At the end of plasma oxidation (i.e. after $10 \mathrm{~s}$ in this case) the intensity of the $2 \mathrm{D}$ peak is reduced (compared to the pristine film) and the $\mathrm{D}$ peak possesses the highest intensity among all Raman peaks. The same trend in Raman spectrum evolution was observed for structural changes of graphene in wet chemical graphene-oxide fabrication [46, 47, 48, 49] and other plasma oxidation studies $[18,19,21,22,23,24,32,33,34,38,39]$. This further confirms gradual transformation of graphene into graphene oxide by oxygen plasma treatment.

As previously discussed, thermal annealing of plasma-treated graphene in an inert atmosphere partially restores $\mathrm{sp}^{2}$ bond symmetry by removing some of the oxygen functionalities which distort the graphene lattice $[20,21,42,43]$. This process is evident in both XPS and Raman results - the $I_{\mathrm{D}} / I_{\mathrm{G}}$ ratio reduces by approximately $25 \%$ after thermal annealing (Figure 2(b)), again, demonstrating that our plasma processing approach is essentially reversible.

In the above we have shown that our blanket CVD graphene films can be successfully transformed, in-situ on $\mathrm{Cu}$ catalytic substrates, into graphene oxide using plasma oxidation. We now demonstrate, via the use of lithographic patterning, that we can selectively oxidize micro- and nano-scale regions of the CVD-grown graphene films. 
(a)

(b)

(c)

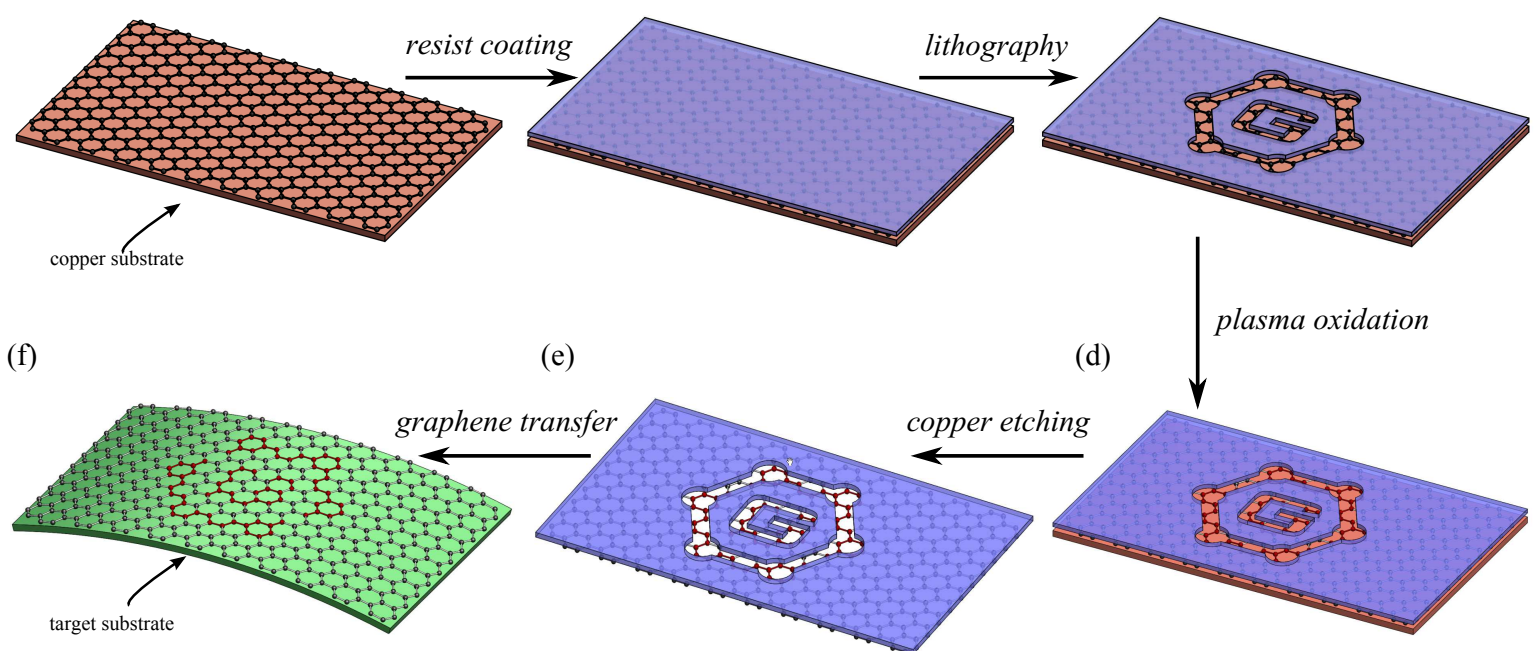

Figure 3. (Colour online) Schematic representation of selective modification of asgrown CVD graphene: (a) to (b) - resist (PMMA) coating; (b) to (c) - e-beam lithography on $\mathrm{Cu}$; (c) to (d) - plasma oxidation (on $\mathrm{Cu}$ ) using RIE system; (d) to (e) - copper etching in ammonium persulfate solution; (e) to (f) - graphene transfer onto the target substrate.

Furthermore, we show that this can also be carried out in-situ directly on the (catalytic) $\mathrm{Cu}$ substrate and in such a way that complete graphene/graphene oxide structures and devices can be fabricated on CVD catalytic metal foils, prior to transfer to a particular target substrate. Our approach is thus compatible with large-area production techniques and provides a simple but very effective in-situ method for creating graphene/GO devices for a range of applications such as non-volatile memories [25, 26], field-effect transistors [50], humidity sensors [30, 31], and even artificial skin [35]. It should be noted that the size-limiting factor of the present work was simply the size of the heating stage of the particular (research-laboratory-scale) CVD growth chamber that we used, which yielded samples of around $16 \mathrm{~cm}^{2}$. However, when combined with wafer-scale [51] or roll-to-roll processing $[52,53,54]$, our technique is scalable right up to the meter size range.

First, to illustrate the flexibility and resolution capabilities of the technique, we selectively modify a graphene film to create a micro-scale $(80$-by- $80 \mu \mathrm{m})$ graphene oxide "G logo" pattern. Next, we use plasma oxidation to treat an array of $100 \mu \mathrm{m}$ squareshaped regions (separated by $100 \mu \mathrm{m}$ gaps) in a continuous graphene film to study capabilities of the $\mathrm{O}_{2}$ plasma oxidation method for larger-scale processing. Finally we produce a graphene/graphene oxide humidity sensor using our selective in-situ plasma oxidation approach.

Our fabrication process is illustrated in Figure 3 and consists of the following stages: (1) a $200 \mathrm{~nm}$ thick layer of positive e-beam resist (PMMA) is spin coated onto graphene film on copper foil, as grown by the CVD method; (2) the area to be functionalized is exposed by e-beam and subsequently developed, leaving the unmasked 
(a)

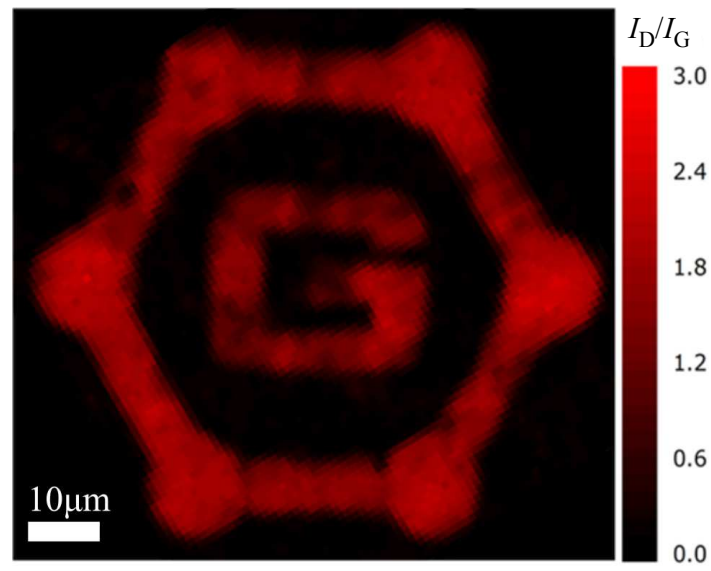

(b)

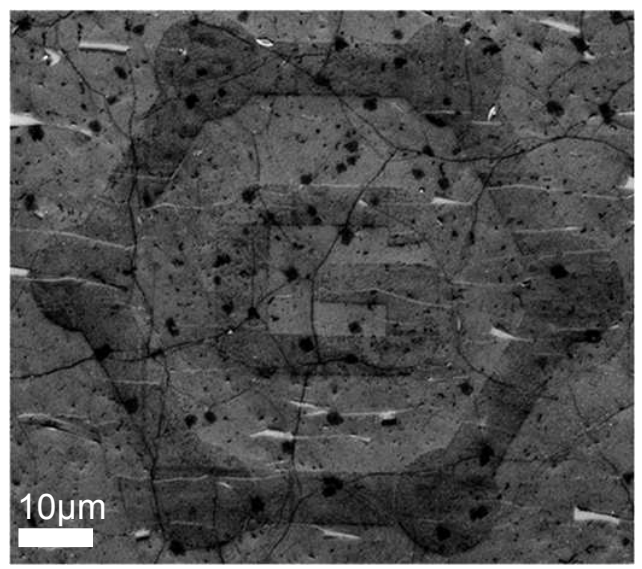

Figure 4. (Colour online) (a) Map of the Raman D-to-G peaks intensities ratio of the selectively oxidized area of continuous CVD graphene film after the transfer onto $\mathrm{Si}$ substrate. (b) SEM image showing change of conductivity in the region exposed to $\mathrm{O}_{2}$ plasma. Multilayer patches (small dark regions) as well as graphene wrinkles (black lines) and PMMA residues (white debris) can be also in the image.

regions of the graphene film exposed; (3) the sample is subjected to an oxygen plasma for functionalization; (4) the copper substrate is etched away from the underside using $0.25 \mathrm{M}$ ammonium persulphate solution; (5) the PMMA-graphene stack is cleaned multiple times in de-ionized water and then transferred onto the target substrate; (6) finally, the PMMA mask is removed using acetone and the sample is cleaned with isopropyl alcohol.

We use optical, Raman and SEM imaging to examine quality of the patterned plasma oxidized structures (see Figure 4). Raman mapping (with $1 \mu \mathrm{m}$ step size) of the $I_{\mathrm{D}} / I_{\mathrm{G}}$ ratio was used to visualize the plasma-exposed area. The $\mathrm{D}$ and $\mathrm{G}$ peaks at each map position were fitted with Lorentzians and the relative intensities were extracted (see Supplementary Material). Figure 4(a) shows the $I_{\mathrm{D}} / I_{\mathrm{G}}$ map for the "G logo" pattern; we notice a striking contrast between the selectively oxidized regions and those that were protected from the plasma. The high resolution of the "G logo" pattern also suggests that a nano-scale degree of control over the plasma oxidation can be achieved with suitably lithographically defined patterns. Indeed, we confirmed such nano-scale patterning capabilities by creating simple, square-shaped window arrays of submicron length and performing Raman measurements on the subsequently plasma oxidized sample (see Supplementary Material, Figure S5). The oxidized "G logo" pattern of Figure 4(a) can also be clearly seen via SEM imaging, as shown in Figure 4(b), the darker color indicating the change of the plasma exposed area from a semi-metallic to semiconductor state $[18,21,22,23,24,32,38,19,55]$. The resulting graphene/graphene oxide structure is of a high quality with no damage such as holes or cracking, as evidenced by the SEM and Raman images. Note also that microscope images (not shown here) of the oxidized areas shows a higher optical transparency of the plasma-exposed regions. The bandgap which is created in graphene due to plasma oxidation was reported to 
(a)

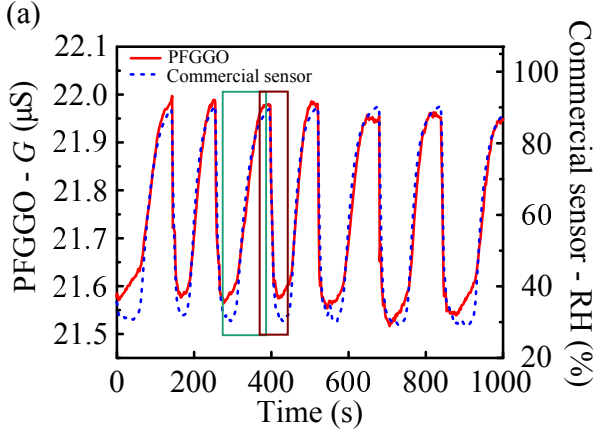

(b)

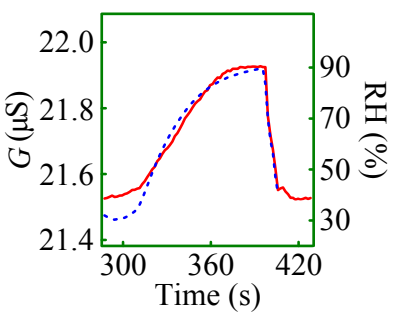

(c)

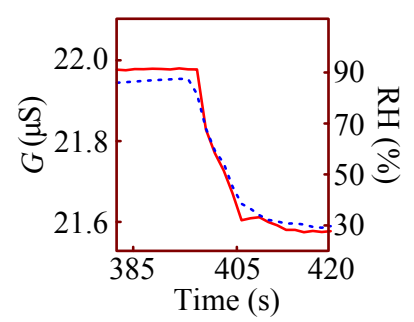

(d)

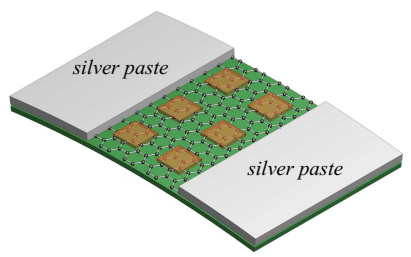

oxidized regions

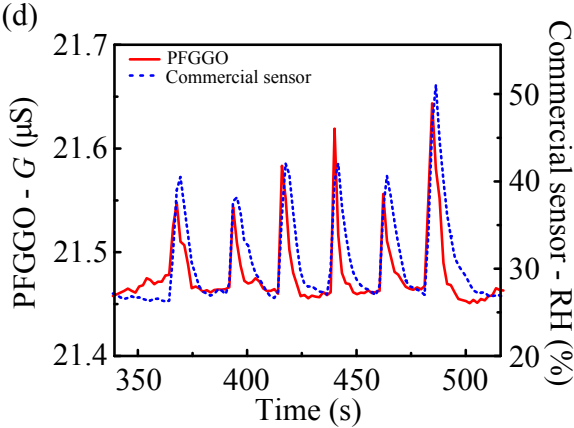

(e)

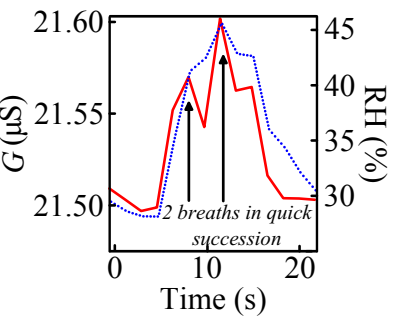

Figure 5. (Colour online) (a) Responses of both sensors upon periodically changing humidity and (b)-(c) zoomed-in rise and fall times of one period (indicated with green and brown rectangles in panel a). (d) Responses of both sensors upon breathing onto devices and (e) responses to the two breaths in quick succession. Schematic representation of the PFGGO humidity sensor is also shown.

be around $2 \mathrm{eV}[18,19,20,55]$ and, thus, oxidation increases film transparency for visible light (a higher visible spectrum transmittance is advantageous for fabricating transparent optoelectronic devices in which visibility of device elements is undesirable).

Next, we demonstrate the direct in-situ device fabrication capabilities of our selective plasma oxidation technique. Since graphene oxide films produced by wet chemistry methods have been shown to possess good properties for sensing environmental humidity [30, 31], it might be expected that plasma oxidized GO materials are also sensitive to humidity. We therefore produced a plasma functionalized graphene/graphene oxide (PFGGO) humidity sensor by selectively oxidizing an array $100 \mu \mathrm{m}$ square-shaped regions (separated by $100 \mu \mathrm{m}$ gaps) in a continuous $1 \mathrm{~cm}$ by $1 \mathrm{~cm}$ CVD graphene film (while still on the catalytic $\mathrm{Cu}$ metal foil). Such an arrangement mimics in a rudimentary way the inter-digitated electrode configuration commonly used in humidity sensing [30, 31]. We transferred the patterned graphene/graphene oxide film to a flexible plastic substrate and, finally, added electrical contacts (using silver paste see schematic of Figure 5) to complete the device. We compared the performance of our directly fabricated PFGGO humidity sensor to a Honeywell HIH-4000-003 calibrated commercial humidity sensor.

The experimental set-up used for the measurements is shown in the Supplementary Material. Both sensors were placed in an environmental chamber within an electrical 
probe station. The analogue output voltage of the commercial sensor was recorded using a Agilent multimeter, while the change in the resistance of the PFGGO sensor was monitored using an LCR bridge. Responses of both devices upon periodically changing the humidity are shown in Figure 5(a); it is clear that the response of PFGGO device follows exactly the humidity variations inside the chamber. The rise and fall times of the PFGGO sensor are also at least as fast as those of the commercial sensor, as we can see in Figures 5(b)-5(c) and where we have "zoomed-in" on a single period of the sensor outputs shown within the green and brown rectangles in Figure 5(a). However, the real response time of both sensors is masked in Figures 5(a)-5(c) by the slowly varying nature of the humidity changes in the environmental chamber. To gain a better indication of the speed of response of the PFGGO sensor we therefore subjected it to a faster change in humidity by breathing on the device. Results of these measurements are presented in Figures 5(d)-5(e) and from which it is clear that the PFGGO sensor was capable of detecting the humidity changes induced by individual breaths during a rapid succession of breaths.

The observed behaviour of the PFGGO sensor can be explained by the interaction of water molecules with the oxygen functionalities of the plasma treated graphene. Water molecules can bind to the introduced oxygen groups to form hydrogen bonds, resulting in the doping of the graphene film $[56,57]$. Such bonding is known to be stronger than Van der Waals physical absorption of water onto the graphene surface and thus provides higher sensitivity to a change in humidity compared to bare graphene films.

\section{Conclusion}

In conclusion we have developed a novel technique for the fabrication of graphene-based devices using in-situ plasma functionalization and in-situ lithographic patterning of graphene directly on (catalytic) CVD metal foil $(\mathrm{Cu})$ substrates. We have proven the concept via the selective plasma oxidation of CVD-graphene so as to produce microand nano-scale graphene/graphene oxide structures and devices that can be transferred in their entirety to a wide range of target substrates (including flexibles, and perhaps even textiles). The structural, chemical, morphological and optical properties of such structures and devices were studied using Raman and X-ray photoelectron spectroscopy and optical and (scanning) electron microscopy. Such studies confirmed the efficacy of the in-situ plasma functionalization approach, the capability for the production of nanoscale functionalized features and the high-quality (uniform, defect-free) nature of the resultant structures and devices. A flexible and transparent graphene/grapheneoxide humidity sensor fabricated using this new approach showed characteristics (in particular a response time) superior to those of a conventional commercial humidity sensor. Although we have concentrated here on oxygen plasma functionalization, our approach is generic and can undoubtedly be applied in the case of functionalization by other chemical species, leading to a wide range of electronic and optoelectronic devices that could be fabricated using our approach. 


\section{Methods}

The CVD-graphene samples were grown using a commercial cold-wall nanoCVD system (supplied by Moorfield Nanotechnology Ltd.) using a "high-pressure" growth recipe provided in Supplementary Material (Table ST1). The growth included two stages, at the first stage graphene nuclei were formed and at the second stage the $\mathrm{CH}_{4}$ proportion was increased to promote formation of a continuous graphene film. After the growth (and, where relevant, in-situ functionalization and patterning), the $\mathrm{Cu}$ foil was etched using $0.25 \mathrm{M}$ ammonium persulfate solution (APS) and graphene films were transferred onto the target substrates using PMMA.

Plasma functionalization of graphene was conducted using commercial reactive ion etching system RIE 80 by JLS Designs Ltd. Mild plasma conditions were used to minimise plasma damage to the graphene backbone: power - $5 \mathrm{~W} ; \mathrm{O}_{2}$ flow - 20 sccm; chamber pressure - $30 \mathrm{mT}$; gas stabilization time - $30 \mathrm{~s}$; treatment duration - 0-20 s; pump out time - $30 \mathrm{~s}$.

The surface chemistry of all graphene films was analyzed using a Kratos AXIS Nova-165 photoelectron spectrometer equipped with a monochromic Al-K $\alpha$ x-ray source $(1486.6 \mathrm{eV})$. All measurements were performed under a high vacuum of $3 \times 10^{-9} \mathrm{mbar}$ at room temperature. The high-resolution XPS C1s spectra (see Figure S2 in Supplementary Material) were collected with a spot size of approx. $400 \mu \mathrm{m}$ and a pass energy of $20 \mathrm{eV}$. Each high-resolution scan collected is an average of 15 scans taken using an energy step of $0.15 \mathrm{eV}$ and a dwell time of $100 \mathrm{~ms}$. In order to identify the chemical composition of species bonded to the graphene film, high-resolution $\mathrm{C} 1 \mathrm{~s}$ spectra were fitted with mixed Gaussian-Lorentzian functions after performing Shirley background subtraction. The chemical nature of all fitted peaks were identified by their relative shift in binding energy with respect to the $\mathrm{C}-\mathrm{C}\left(\mathrm{sp}^{2}\right)$ peak. A quantification scaling was performed, where all spectra were normalized with respect to the intensity of unoxidized C1s peak.

Raman measurements were performed using a Renishaw Raman RM1000 and Horiba Xplora systems fitted with $532 \mathrm{~nm}$ excitation sources and air-cooled chargecoupled detectors. Raman signals were collected by focusing the laser onto the sample to a spot size of approx $1.0 \mu \mathrm{m}$ to approx $1.5 \mu \mathrm{m}$ at less than $2 \mathrm{~mW}$ of power to avoid laser induced damage. Lorentzian peak fitting (as shown in Figure S3 in Supplementary Material) was performed to precisely determine the positions, full-width at half maximum (FWHM), and intensities of the Raman peaks in the $I_{\mathrm{D}} / I_{\mathrm{G}}$ mapping of the "G logo" (see main text). For each map position the scan was fitted for D, G and $\mathrm{D}^{\prime}$ peaks with a multiple Lorentzian and the peak parameters were extracted. The stage was scanned in (roughly) $1 \mu \mathrm{m}$ steps over the area of the "G logo".

Humidity measurements were performed using a probe station with environmental chamber and against commercial humidity sensor HIH-4000-03 by Honeywell. Humidity inside the probe station chamber was varied in a periodic manner and responses of the commercial sensor and our PFGGO sensor were recorded simultaneously. The 
commercial humidity sensor was powered using a Keithley 2400 meter (DC voltage, $5 \mathrm{~V}$ ) while our PFGGO sensor was connected to a Rohde \& Schwarz HM8118 LCR bridge $(500 \mathrm{~Hz}, 0.5 \mathrm{~V})$. The experimental set-up (probe station with environmental chamber) is shown in Figure S6 in the Supplementary Material.

\section{Acknowledgments}

This work was carried out under the auspices of the EU FP7 project CareRAMM. This project received funding from the European Union Seventh Framework Programme (FP7/2007-2013) under grant agreement no 309980. The authors are grateful for helpful discussions with all CareRAMM partners, particularly Prof A. Ferrari and Ms A. Ott at the University of Cambridge, and Dr A. Sebastian and Dr W. Koelmans at IBM Research Zurich. We also gratefully acknowledge the assistance of the National EPSRC XPS User's Service (NEXUS) at Newcastle University, UK (an EPSRC Mid-Range Facility) in carrying out the XPS measurements and the assistance of Prof S. Russo at the University of Exeter in carrying out humidity sensing measurements.

A.M.A. would also like to thank Dr E. Alexeev for useful ideas for this Letter and pleasurable discussions of the results.

\section{References}

[1] Kang J, Shin D, Bae S, Hong B H 2012 Nanoscale 4 5527-5537.

[2] Iqbal M Z, Singh A K, Iqbal M W, Seo S, Eom J 2012 J. Appl. Phys. 111084307.

[3] Shen X, Wang H, Yu T 2013 Nanoscale 5 3352-3358.

[4] Gong C, McDonnell S, Qin,X, Azcatl A, Dong H, Chabal Y J, Cho K, Wallace R M 2014 ACS Nano 8 642-649.

[5] Robinson J A, LaBella M, Zhu M, Hollander M, Kasarda R, Hughes Z, Trumbull K, Cavalero R, Snyder D 2011 Appl. Phys. Lett. 98053103.

[6] Smith J T, Franklin A D, Farmer D B 2013 ACS Nano 7 3661-3667.

[7] Asadi K, Timmering E C, Geuns T C, Pesquera A, Centeno A, Zurutuza A, Klootwijk J H, Blom P W, de Leeuw D M 2015 ACS Appl. Mater. Interfaces 7 9429-9435.

[8] Robinson J T, Burgess J S, Junkermeier C E, Badescu S C, Reinecke T L, Perkins F K, Zalalutdniov M K, Baldwin J W, Culbertson J C, Sheehan P E, Snow E S 2010 Nano Lett. 10 3001-3005.

[9] Withers F, Russo S, Dubois M, Craciun M F 2011 Nanoscale Res. Lett. 6 1-11.

[10] Craciun M F, Khrapach I, Barnes M D, Russo S 2013 J. Phys.: Condens. Matter 25423201.

[11] Lee W H, Suk J W, Lee J, Hao Y, Park J, Yang J W, Ha H W, Murali S, Chou H, Akinwande D, Kim K S 2012 ACS Nano 6 1284-1290.

[12] Lee W H, Suk J W, Chou H, Lee J, Hao Y, Wu Y, Piner R, Akinwande D, Kim K S, Ruoff R S 2012 Nano Lett. 12 2374-2378.

[13] Sahin H, Ciraci S 2012 J. Phys. Chem. C 116 24075-24083.

[14] Karlicky F, Kumara Ramanatha Datta K, Otyepka M, Zboril R 2013 ACS Nano 7 6434-6464.

[15] Kim N, Kim K S, Jung N, Brus L, Kim P 2011 Nano Lett. 11 860-865.

[16] Khrapach I, Withers F, Bointon T H, Polyushkin D K, Barnes W L, Russo S, Craciun M F 2012 Adv. Mater. 24 2844-2849.

[17] Wehenkel D J, Bointon T H, Booth T, Boggild P, Craciun M F, Russo S 2015 Sci. Rep. 57609.

[18] Gokus T, Nair R R, Bonetti A, Bohmler M, Lombardo A, Novoselov K S, Geim A K, Ferrari A C, Hartschuh A 2009 ACS Nano 3 3963-3968. 
[19] Nourbakhsh A, Cantoro M, Vosch T, Pourtois G, Clemente F, van der Veen M H, Hofkens J, Heyns M M, de Gendt S, Sels B F 2010 Nanotechnology 21435203.

[20] Aria A I, Gani A W, Gharib M 2014 Appl. Surf. Sci. 293 1-111.

[21] Baraket M, Walton S G, Lock E H, Robinson J T, Perkins F K 2010 Appl. Phys. Lett. 96231501.

[22] Huang C-H, Su C-Y, Lai C-S, Li Y-C, Samukawa S 2014 Carbon 73 244-251.

[23] Liu L, Xie D, Wu M, Yang X, Xu Z, Wang W, Wang E 2012 Carbon 50 3039-3044.

[24] McEvoy N, Nolan H, Ashok Kumar N, Hallam T, Duesberg G S 2013 Carbon 54 283-290.

[25] Jeong H Y, Kim J Y, Kim J W, Hwang J O, Kim J E, Lee J Y, Yoon T H, Cho B J, Kim S O, Ruoff R S, Choi S Y 2010 Nano Lett. 10 4381-4386.

[26] Liu J, Yin Z, Cao X, Zhao F, Wang L, Huang W, Zhang H 2013 Adv. Mater. 25 233-238.

[27] Porro S, Accornero E, Pirri C F, Ricciardi C 2015 Carbon 85 383-396.

[28] Ogata C, Kurogi R, Hatakeyama K, Taniguchi T, Koinuma M, Matsumoto Y 2016 Chem. Commun. 52 3919-3922.

[29] Prezioso S, Perrozzi F, Giancaterini L, Cantalini,C, Treossi E, Palermo V, Nardone M, Santucci S, Ottaviano L 2013 J. Phys. Chem. C 117 10683-10690.

[30] Bi H, Yin K, Xie X, Ji J, Wan S, Sun L, Dresselhaus M S 2013 Sci. Rep. 32714.

[31] Borini S, White R, Wei D, Astley M, Haque S, Spigone E, Harris N, Kivioja J, Ryhanen T 2013 ACS Nano 7 11166-11173.

[32] Felten A, Flavel B S, Britnell L, Eckmann A, Louette P, Pireaux J J, Hirtz M, Krupke R, Casiraghi C 2013 Small 9631

[33] Marsden A J, Brommer P, Mudd J J, Dyson M A, Cook R, Asensio M, Avila J, Levy A, Sloan J, Quigley D, Bell G R 2015 Nano Res. 8 2620-2635.

[34] Santoso I, Singh R S, Gogoi P K, Asmara T C, Wei D, Chen W, Wee A T, Pereira V M, and Rusydi A 2014 Phys. Rev. B 89 1-9.

[35] Bointon T H, Barnes M D, Russo S, Craciun M F 2015 Adv. Mater. 27 4200-4206.

[36] Tao L, Lee J, Chou H, Holt M, Ruoff R S, Akinwande D 2012 ACS Nano 6 2319-2325.

[37] Huang L, Chang Q H, Guo G L, Liu Y, Xie Y Q, Wang T, Ling B, Yang H F 2012 Carbon 50 $551-556$.

[38] Foley B M, Hernandez S C, Duda J C, Robinson J T, Walton S G, Hopkins P E 2015 Nano Lett. 15 4876-4882.

[39] Mulyana Y, Uenuma M, Ishikawa Y, Uraoka Y 2009 J. Phys. Chem. C 118 27372-27381.

[40] Kidambi P R, Bayer B C, Blume R, Wang Z J, Baehtz C, Weatherup R S, Willinger M G, Schloegl R, Hofmann S 2013 Nano Lett. 13 4769-4778.

[41] Blume R, Kidambi P R, Bayer B C, Weatherup R S, Wang Z J, Weinberg G, Willinger M G, Greiner M, Hofmann S, Knop-Gericke A, Schlogl R 2014 Phys. Chem. Chem. Phys. 16 2598926003.

[42] Bagri A, Mattevi C, Acik M, Chabal Y J, Chhowalla M, Shenoy V B 2010 Nat. Chem. 2 581-587.

[43] Vinogradov N A, Schulte K, Ng M L, Mikkelsen A, Lundgren E, Martensson N, Preobrajenski A B 2011 J. Phys. Chem. C 115 9568-9577.

[44] Tuinstra F and Koenig J L 1970 J. Chem. Phys. 53 1126-1130.

[45] Ferrari A C and Basko D M 2013 Nat. Nanotech. 8 235-246.

[46] Dreyer D R, Park S, Bielawski C W, Ruoff R S 2010 Che. Soc. Rev. 39 228-240.

[47] Zhu Y, Murali S, Cai W, Li X, Suk J W, Potts J R, Ruoff R S 2010 Adv. Mater. 22 3906-3924.

[48] Chen D, Feng H, Li J 2012 Chem. Rev. 112 6027-6053.

[49] Kudin K N, Ozbas B, Schniepp H C, Prud'Homme R K, Aksay I A, Car R 2008 Nano Lett. 8 $36-41$.

[50] Schwierz F 2010 Nat. Nanotech. 5 487-496.

[51] Gao L, Ni G X, Liu Y, Liu B, Neto A H C, Loh K P 2014 Nature, 505 190-194.

[52] Bae S, Kim H, Lee Y, Xu X, Park J S, Zheng Y, Balakrishnan J, Lei T, Kim H R, Song Y I, Kim Y J 2010 Nat. Nanotech. 5 574-578.

[53] Ahn S H and Guo L J 2008 Adv. Mater. 20, 2044-2049. 
[54] Cheng T S and Lin H T, Chuang M J 2004 Mater. Lett. 58 650-653.

[55] Nourbakhsh A, Cantoro M, Klekachev A V, Pourtois G, Vosch T, Hofkens J, van der Veen M H, Heyns M M, de Gendt S, Sels B F 2011 J. Phys. Chem. C 115 16619-16624.

[56] Hao Y, Meng G, Ye C, Zhang L 2005 Appl. Phys. Lett. 87033106.

[57] Guo L, Jiang H B, Shao R Q, Zhang Y L, Xie S Y, Wang J N, Li X B, Jiang F, Chen Q D, Zhang T, Sun H B 2012 Carbon 50 1667-1673. 